\title{
MODELO DISCRETO CIERTO (Y SIMPLE) PARA DETERMINAR UNA RATIO DE PÉRDIDAS POR CATÁSTROFES SUBYACENTE DE LOS DERIVADOS SOBRE SEGUROS (INSURANCE-LINKED DERIVATIVES, ILS)*
}

\section{A SIMPLE DISCRETE MODEL TO CALCULATE CATASTROPHIC LOSS INDEX TRIGGERS FOR INSURANCE-LINKED DERIVATIVES}

\author{
María José Pérez-Fructuoso** \\ Fecha de recepción: 7 de Junio de 2016 \\ Fecha de Aceptación: 15 de julio de 2016 \\ Disponible en línea: 30 de Julio de 2016
}

\section{Para Citar este articulo/To cite this article}

Pérez-Fructuoso, María José, Modelo discreto cierto (y simple) para determinar una ratio de pérdidas por catástrofes subyacente de los derivados sobre seguros (insurance-linked derivatives, ils), 44 Rev.Ibero-Latinoam.Seguros, 185-205 (2016). http://dx.doi.org/10. 11144/Javeriana.ris44.mdcd doi:10.11144/Javeriana.ris44.mded

* Artículo de investigación que desarrolla un modelo discreto simple para determinar un índice de pérdidas por catástrofes.

** Doctora Europera en Economía, Doctora en Ciencias Económicas y Empresariales. Licenciada en Ciencias Actuariales y Financieras. Licenciada en Ciencias Económicas y Empresariales. Profesora Titular del Área de Economía Financiera y Contabilidad, Madrid Open University (UDIMA). Contacto: mariajose.perez@udima.es 


\section{RESUMEN}

En este artículo se desarrolla un modelo discreto simple para determinar un índice de pérdidas por catástrofes que pueda utilizarse como subyacente de los derivados vinculados a seguros (insurance-linked derivatives). Para ello se considera que en cada periodo únicamente puede producirse una catástrofe cuya cuantía total está formada por la suma de dos variables: la cuantía de siniestros pendiente de declarar y la cuantía declarada de siniestros. Como hipótesis central del modelo se supone que la cuantía de siniestros pendiente de declarar decrece proporcionalmente en el tiempo a razón de una tasa constante, denominada "tasa nominal de declaración de siniestros", que, en el caso de periodos unitarios, coincide con la tasa efectiva de declaración de siniestros y que funciona como una tasa nominal de descuento.

Palabras clave: cuantía de siniestros declarada; cuantía de siniestros pendiente de declarar; índice de pérdidas; tasa nominal de declaración de siniestros. 


\section{ABSTRACT}

This paper developes a simple discrete model to determine catastrophic loss indexes underlying insurance-linked derivatives. To this aim, we consider just a single catastrophe ocurring in each interval, whose total amount results from the sum of two variables: the incurred-but-not-yet-reported claims amount and that of the reported claims. Our model's core assumption is that the incurredbut-not-yet-reported claims amount decreases proportionally in time at the rate of a constant named "nominal claim reporting rate," which coincides with the effective claim reporting rate in the case of unitary periods, and which works as a nominal rate of discount.

Key words: incurred-but-not-yet-reported loss amount; reported loss amount; asymptotic claim reporting rate; catastrophic loss index; nominal claim reporting rate.

\section{SUMARIO}

1. INTRODUCCIÓN. 2. DEFINICIONES GENERALES DEL MODELO 2.1. Hipótesis básicas sobre la ocurrencia de las catástrofes. 2.2. Hipótesis básicas sobre la declaración de los siniestros. 3. DETERMINACIÓN DE LA DINÁMICA DE LA RATIO DE SINIESTRALIDAD. 4. CONCRECIÓN DEL MODELO. SUSTITUCIÓN DE LOS INCREMENTOS $\Delta_{\varphi} Y_{\tau} .5$. CONCLUSIONES. BIBLIOGRAFÍA. 


\section{INTRODUCCIÓN}

La investigación que se desarrolla en el presenta artículo versa sobre un tipo de activos financieros, los contratos de activos derivados sobre riesgos catastróficos, de aparición relativamente reciente en el tiempo. Su aparición en el escenario financiero y actuarial data tan sólo de 1992. Hasta la década de los 60, las aseguradoras norteamericanas empleaban los contratos tradicionales de reaseguro como instrumento para afrontar la siniestralidad derivada de los riesgos de naturaleza catastrófica. A partir de 1970 y hasta 1992, la mayoría de estos grandes eventos de suceso imprevisible y de graves consecuencias económicas no llegaron a alcanzar los 250 millones de dólares en pérdidas, razón por la cual pudo ser hasta entonces el reaseguro, mecanismo tradicional para la cobertura de riesgos catastróficos, cauce técnico suficiente para canalizar este tipo de operaciones.

1992 marcó, sin embargo, un punto de inflexión en hasta entonces esta línea ordinaria de actuación de las compañías del ramo: en ese año concurrieron varios factores, cuya acumulación provocó la insuficiencia del reaseguro como procedimiento idóneo para la cobertura de este tipo de riesgos. Por una parte, algunas grandes reaseguradoras sufrieron importantes dificultades financieras. Por otra, se dio la circunstancia especial de que se sucedieron dos grandes catástrofes en un corto periodo de tiempo: durante el tercer trimestre de 1992, el huracán Andrew dejó un abultado balance de 18.600 millones de dólares en pérdidas. Escasas fechas después, los daños causados por el huracán Iniki alcanzan una cifra aproximada de 1.600 millones de dólares.

La gran magnitud de estas dos catástrofes, y su casi coincidencia en el tiempo tuvieron una honda repercusión sobre la actividad de las reaseguradoras norteamericanas: la reducción de sus límites de aceptación en riesgos de este tipo. Como efecto derivado, las compañías de seguros se vieron fuertemente incentivadas para la búsqueda de nuevos sistemas de dispersión del riesgo. Así, el Chicago Board of Trade lanzó en diciembre de ese mismo año el primer instrumento de cobertura diseñado específicamente para la industria del seguro: los contratos de futuros y de opciones sobre riesgos catastróficos, CAT-futures y opciones CAT. Los contratos CAT fueron substituidos en septiembre de 1995 por las opciones PCS por falta de negociación en los mercados. 
Sin embargo, los productos financieros derivados más desarrollados hasta la fecha, han sido los Bonos sobre Catástrofes (o Cat bonds). Estos activos condicionan los flujos de capital, cupones y principal, a la ocurrencia de un determinado suceso de naturaleza catastrófica al que se conoce como trigger o desencadenante y que suele ser un índice de pérdidas por su mayor simplicidad interpretativa.

En una aproximación teórica, una de las facetas principales en el análisis de estos activos derivados es su valoración a lo largo de un intervalo temporal determinado. Para ello, es preciso desarrollar un modelo que permita calcular la evolución en el tiempo de la suma total de las pérdidas, $L I\left(T^{\prime}\right)$, y, obviamente, de la ratio de siniestralidad subyacente de ambos géneros de contratos.

Diversos autores han abordado el problema de la valoración de estos activos derivados. El método habitualmente empleado se asienta sobre el desarrollo de modelos de tarificación continuos basados en la hipótesis de movimiento browniano geométrico, cuyo propósito es sistematizar la evolución de la declaración instantánea de los siniestros, y dar cabida en dichos modelos, a través de procesos de Poisson, a la posibilidad de que sobrevengan grandes catástrofes. Algunos ejemplos de estos modelos continuos son los desarrollados por Cummins y Geman (1995); Geman y Yor, (1997); Embrechts y Meister (1997); Aase (1999 y 2001); Cox y Pedersen (2000); Baryshnikov, Mayo y Taylor (2001); Lee y Yu (2002); Alegre, Pérez-Fructuoso y Devolder (2003); Burnecki y Kukla (2003); Muermann (2003); Burnecki (2005); Lee y Yu (2007); Egami y Young (2008); Pérez-Fructuoso (2008 y 2009); Biagini, Bregman y Meyer-Brandis (2008); Unger (2010); Nowak y Romaniuk (2013); Zong-Gang y Chao-Qun (2013) y Lai, Parcollet y Lamond (2014).

Es práctica consolidada entre los círculos actuariales valorar las declaraciones de siniestros, tales como las contenidas en el subyacente de los contratos de activos derivados sobre catástrofes, como la suma de dos variables aleatorias: el número de siniestros, por una parte, y la cuantía o coste de cada siniestro, por otra, conforme a la expresión:

$$
C=\sum_{i=1}^{N(t)} X_{i}
$$

siendo $C$ la siniestralidad total, $N(t)$ el número de siniestros y $X_{i}$ la cuantía de cada siniestro (Panjer, H., 1998). 
La modelación del número de siniestros se realiza a través de distribuciones de probabilidad discretas, Poisson o Binomial negativa. La cuantía de cada siniestro, por su parte, queda representada mediante distribuciones continuas como la distribución Gamma, Lognormal o Pareto. Además, se ha asumido que las variables aleatoria $X_{i}$ son independientes entre sí e idénticamente distribuidas e independientes de la variable aleatoria número de siniestros.

A su vez, conocidas las funciones de distribución del número de siniestros y de la cuantía de cada uno de ellos, se establece la distribución de la siniestralidad total, $C$; dicho de otro modo, sobre la base de tales datos se obtiene la probabilidad de que la siniestralidad total sea menor o igual que un determinado valor, $x$, condicionada a que el número de siniestros ocurridos tome el valor $n$.

Si llamamos $F_{X}^{k^{*}}(x)$ a la convolución $k$-ésima de la distribución de probabilidad de la cuantía de cada siniestro y $p[N(t)=n]$ a la distribución de probabilidad de Poisson, la función de distribución de la siniestralidad total resulta,

$$
F(x)=\sum_{n=1}^{\infty} p[N(t)=n] \cdot F_{X}^{k *}(x)
$$

expresión que, según es conocido, se denomina como 'función de distribución compuesta de Poisson" (Latorre, L. ,1992).

El precio del seguro basado en estos modelos se calcula de manera habitual hallando la esperanza matemática de la distribución de la siniestralidad total y añadiéndole a dicha esperanza un recargo de seguridad, normalmente dependiente del segundo momento de la distribución. Sin embargo, este procedimiento de determinación del precio del seguro es incongruente con la valoración realizada en los mercados financieros, en los que, la cuantificación de los activos derivados se obtiene, evitando las oportunidades de arbitraje, por la vía de la réplica de carteras formadas por activos simples cuyos resultados son iguales en todo momento del periodo de negociación al del activo derivado objeto de valoración.

Partiendo del análisis empírico de la evolución de las declaraciones de siniestros después de suceder una catástrofe, en este artículo se propone un modelo matemático en tiempo discreto para calcular la componente aleatoria de la ratio de siniestralidad subyacente de esta clase de activos derivados. 
Para formalizar el modelo consideramos que la cuantía total de una catástrofe es la suma de la cuantía de siniestros declarada y de la cuantía de siniestros pendiente de declarar, reduciendo la posibilidad de ocurrencia de catástrofes a una por periodo. El modelo discreto es objeto de planteamiento, con apoyo de un enfoque determinista: se supone que el decrecimiento temporal de la cuantía de siniestros pendiente de declarar guarda relación proporcional con un valor constante, que denominamos tasa nominal de declaración de siniestros. La dinámica de la cuantía de siniestros pendiente de declarar queda definida entonces a través de una ecuación en diferencias finitas cierta.

El artículo está estructurado de la siguiente forma. En la sección 2, tras la definición de las hipótesis básicas sobre las que se modela la ocurrencia de las catástrofes y la declaración de los siniestros, se presenta las soluciones de las variables cuantía declarada de siniestros y cuantía de siniestros pendientes de declarar utilizando una metodología inductiva y para un valor genérico de los incrementos de la cuantía declarada de siniestros. La Sección 3 se dedica al cálculo del índice de pérdidas por catástrofes, a partir de los resultados obtenidos en la Sección anterior. En la Sección 4 concretamos el modelo, asignando una ecuación en diferencias finitas cierta para modelar la dinámica discreta de la cuantía de siniestros pendiente de declarar. Finalmente, la Sección 5 presenta las principales conclusiones alcanzadas en el trabajo.

\section{DEFINICIONES GENERALES DEL MODELO}

\subsection{Hipótesis básicas sobre la ocurrencia de las catástrofes}

Suponemos que $[0, T] \subset\left[0, T^{\prime}\right]$ es el periodo de riesgo del derivado sobre seguros, tal que $T^{\prime} \geq T$ es la fecha de vencimiento o amortización del contrato.

Hacemos que $K_{\tau}$ con $\tau \in\{1,2, \ldots, T\}$ sea el volumen total de la catástrofe ocurrida durante el periodo $[\tau-1, \tau]$, y $\tau \in\{1,2, \ldots, T\}$ los momentos en los que puede producirse las catástrofes de cuantía $K_{\tau}$. Además se considera que esta cuantía es la suma de dos variables, la cuantía declarada de siniestros hasta $t$ y la cuantía de siniestros pendiente de declarar en $t$ :

$$
K_{\tau}=S_{\tau}(t)+R_{\tau}(t)
$$


Por último, en el periodo de pérdidas, definimos la variable aleatoria dicotómica $\delta_{\tau}$ como aquella variable que da, en cada periodo, la probabilidad de ocurrencia de una catástrofe. Esta variable aleatoria puede tomar dos valores; 0 si no se produce la catástrofe en el momento $\tau$ con probabilidad $p \quad$ y 1 si se produce la catástrofe en el momento $\tau$ con probabilidad $1-p$.

\subsection{Hipótesis básicas sobre la declaración de los siniestros}

Definimos $\varphi \in\left\{\tau, \tau+1, \ldots, T, T+1, \ldots, t, \ldots T^{\prime}\right\}$ como los posibles momentos de valoración considerados a partir del momento en el que se produce una catástrofe, siendo $\varphi=t$ un momento de valoración genérico y suponemos que en $\varphi=1$ se inicia la observación de la evolución de las declaraciones de siniestros asociadas a la ocurrencia de catástrofes.

Utilizando una metodología inductiva, obtenemos las expresiones que nos permiten calcular la cuantía declarada de siniestros en el momento $t$, y la cuantía de siniestros pendiente de declarar en ese momento, $S_{\tau}(t)$ y $R_{\tau}(t)$ respectivamente, variables fundamentales para la determinación de la ratio de siniestralidad objeto de estudio.

Partiendo de esta base, transcurrido el primer periodo y situados en el momento $\tau=1$ pueden darse dos situaciones:

Situación 1: No se produce ninguna catástrofe.

Situación 2: Se produce una catástrofe de cuantía total $K_{1}$.

Si al final del primer periodo se da la Situación 2, suponemos que, de la cuantía total de la catástrofe ocurrida, $K_{1}$ se declara una parte, $Y_{1}$, conocida en ese momento.

Por tanto, si valoramos en $t=1$ tenemos,

$$
K_{1}=S_{1}(1)+R_{1}(1)
$$

donde la cuantía declarada de siniestros hasta el momento de valoración, $t=1$, asociada a la catástrofe ocurrida al final del periodo del primer periodo vale,

$$
S_{1}(1)=Y_{1}
$$


y la cuantía de siniestros pendiente de declarar es:

$$
R_{1}(1)=K_{1}-S_{1}(1)=K_{1}-Y_{1}
$$

Transcurrido el siguiente periodo y situados en el momento $\tau=2$ pueden darse cuatro situaciones:

Situación 1: durante el primer periodo no se ha producido ninguna catástrofe y durante el segundo periodo no se produce ninguna catástrofe.

Situación 2: durante el primer periodo no se ha producido ninguna catástrofe y durante el segundo periodo se produce una catástrofe de cuantía total $K_{2}$ de la cual se declara una parte, $Y_{2}$ conocida en ese momento.

En este caso, el total acumulado de declaraciones de siniestros, si valoramos en $t=2$, correspondientes a las catástrofes ocurridas en $\tau=\{1,2\}$ es:

$$
S_{2}(2)=Y_{2}
$$

y la cuantía de siniestros pendiente de declarar es:

$$
R_{2}(2)=K_{2}-S_{2}(2)=K_{2}-Y_{2}
$$

Situación 3: durante el primer periodo se produce una catástrofe de cuantía total $K_{1}$ y durante el segundo periodo no se produce ninguna catástrofe.

En este caso, al final del segundo periodo se declara una parte determinista, $\Delta_{1} Y_{1}$, de la catástrofe ocurrida en el primer periodo. El total acumulado de declaraciones de siniestros, si valoramos en $t=2$, asociado a las catástrofes ocurridas en $\tau=\{1,2\}$ vale, por tanto,

$$
S_{1}(2)=Y_{1}+\Delta_{1} Y_{1}
$$

y la cuantía de siniestros pendiente de declarar es:

$$
R_{1}(2)=K_{1}-S_{1}(2)=K_{1}-\left(Y_{1}+\Delta_{1} Y_{1}\right)
$$

Situación 4: durante el primer periodo se produce una catástrofe de cuantía total $K_{1}$ y durante el segundo periodo se produce una catástrofe de cuantía total $K_{2}$ de la cual se declara una parte, $Y_{2}$, conocida en ese momento.

En este caso, al final del segundo periodo se declara una parte determinista, $\Delta_{1} Y_{1}$, de la catástrofe ocurrida en el primer periodo. El total 
acumulado de declaraciones de siniestros, si valoramos en $t=2$, asociado a las catástrofes ocurridas en $\tau=\{1,2\}$ vale, por tanto,

$$
\begin{gathered}
S_{1}(2)=Y_{1}+\Delta_{1} Y_{1} \\
S_{2}(2)=Y_{2}
\end{gathered}
$$

y la cuantía de siniestros pendiente de declarar es:

$$
\begin{gathered}
R_{1}(2)=K_{1}-\left(Y_{1}+\Delta_{1} Y_{1}\right) \\
R_{2}(2)=K_{2}-Y_{2}
\end{gathered}
$$

En general, transcurridos $t$ periodos y situados en dicho momento, encontramos $2^{t}$ situaciones en función de la posibilidad de ocurrencia de catástrofes en cada periodo considerado. Si valoramos en ese momento, la cuantía declarada de siniestros asociada a la catástrofe ocurrida en el momento $\tau$ resulta:

$$
S_{\tau}(t)=\delta(\tau)\left[\sum_{\varphi=0}^{t-\tau} \Delta_{\varphi} Y_{\tau}\right] \operatorname{con} \Delta_{0} Y_{\tau}=Y_{\tau}
$$

Sustituyendo la expresión (2) en la igualdad (1) y despejando, obtenemos la expresión para la cuantía de siniestros pendiente de declarar en $t$ asociada a la ocurrencia de la catástrofe en el momento $\tau, R_{\tau}(t)$ :

$$
R_{\tau}(t)=\delta(\tau)\left[K_{\tau}-\sum_{\varphi=0}^{t-\tau} \Delta_{\varphi} Y_{\tau}\right] \operatorname{con} \Delta_{0} Y_{\tau}=Y_{\tau}
$$

\section{DETERMINACIÓN DE LA DINÁMICA DE LA RATIO DE SINIESTRALIDAD}

La ratio de siniestralidad subyacente de los contratos de activos derivados sobre riesgos catastróficos se define como,

$$
L I\left(T^{\prime}\right)=\sum_{\tau=1}^{T^{\prime}} \frac{S_{\tau}\left(T^{\prime}\right)}{c t e}
$$


donde el numerador es la cuantía total de las declaraciones de pérdidas en el momento $T^{\prime}$ asociadas a la ocurrencia de catástrofes durante el periodo de pérdidas.

Entonces, tomando la expresión (2) y sustituyéndola en el sumatorio (4), la cuantía total de pérdidas en $T^{\prime}, L I\left(T^{\prime}\right)$, resulta:

$$
L I\left(T^{\prime}\right)=\frac{1}{c t e} \sum_{\tau=1}^{T^{\prime}} S_{\tau}\left(T^{\prime}\right)=\frac{1}{c t e} \sum_{\tau=1}^{T} \delta(\tau)\left[\sum_{\varphi=0}^{T^{\prime}-\tau} \Delta_{\varphi} Y_{\tau}\right]
$$

Transcurridos $t$ periodos, y situados en el momento de valoración $\varphi=t$ disponemos de la información acerca de todas las catástrofes ocurridas y de todos los siniestros declarados hasta ese momento. Teniendo en cuenta estos datos, definimos una nueva variable aleatoria que simbolizamos como $L I\left(T^{\prime}\right) \mid F_{t}$. Esta variable aleatoria condicionada incorpora, a través de la filtración $F_{t}$ la historia pasada sobre las catástrofes, ocurridas y declaradas, hasta el momento de valoración.

El primer paso para obtener la variable aleatoria condicionada, $L I\left(T^{\prime}\right) \mid F_{t}$, es calcular su condicionante dado por la cuantía total de las pérdidas declaradas hasta el momento $t$ de valoración $L I(t)$. Entonces, considerando la existencia de dos periodos diferenciados en cuanto a la formación de la ratio de siniestralidad, tenemos que:

- Si $t \in\{1,2, \ldots, T\}, L I(t)$ hace referencia a las pérdidas declaradas hasta el momento $t$, asociadas a las catástrofes que hayan ocurrido hasta ese momento:

$$
L I(t)=\frac{1}{c t e} \sum_{\tau=1}^{t} S_{\tau}(t)=\frac{1}{c t e} \sum_{\tau=1}^{t} \delta(\tau)\left[\sum_{\varphi=0}^{t-\tau} \Delta_{\varphi} Y_{\tau}\right]
$$

- Si $t \in\left\{T+1, T+2, \ldots, T^{\prime}\right\}, L I(t)$ hace referencia a las pérdidas declaradas hasta el momento $t$, asociadas a las catástrofes que hayan ocurrido hasta el final del periodo de riesgo, $T$ :

$$
L I(t)=\frac{1}{c t e} \sum_{\tau=1}^{T} S_{\tau}(t)=\frac{1}{c t e} \sum_{\tau=1}^{T} \delta(\tau)\left[\sum_{\varphi=0}^{t-\tau} \Delta_{\varphi} Y_{\tau}\right]
$$


En general, para cualquier momento $t \in\left\{1,2, \ldots, T, T+1, T+2, \ldots, T^{\prime}\right\}$, la expresión para la cuantía $L I(t)$ resulta:

$$
L I(t)=\frac{1}{c t e} \sum_{\tau=1}^{\min \{t, T\}} S_{\tau}(t)=\frac{1}{c t e} \sum_{\tau=1}^{\min \{t, T\}} \delta(\tau)\left[\sum_{\varphi=0}^{t-\tau} \Delta_{\varphi} Y_{\tau}\right]
$$

Obtenida la cuantía $L I(t)$, la introducimos en la variable $L I\left(T^{\prime}\right)$, descomponiendo la variable aleatoria condicionada, $\operatorname{LI}\left(T^{\prime}\right) \mid F_{t}$, en la suma de dos componentes tal que:

$$
\begin{aligned}
& \text { Si } t \in\{1,2, \ldots, T\}, \\
& L I\left(T^{\prime}\right) \mid F_{t}=L I_{1}\left(T^{\prime}\right)+L I_{2}\left(T^{\prime}\right)
\end{aligned}
$$

donde $L I_{1}\left(T^{\prime}\right)$ es la cuantía de pérdidas declaradas hasta el momento $T^{\prime}$ que incorpora la información disponible sobre las catástrofes ocurridas y declaradas hasta el momento $t$ y $L I_{2}\left(T^{\prime}\right)$ es la cuantía de pérdidas declaradas hasta el momento $T^{\prime}$ asociada a las catástrofes ocurridas después del momento $t$ y hasta $T$.

La componente $L I_{1}\left(T^{\prime}\right)$ de la variable condicionada la calculamos a partir del total acumulado de declaraciones en $t, L I(t)$ como sigue,

$$
L I_{1}\left(T^{\prime}\right)=\frac{1}{c t e} \sum_{\tau=1}^{t}\left(\delta(\tau) \mid F_{t}\right)\left\{\sum_{\varphi=0}^{T^{\prime}-\tau} \Delta_{\varphi} Y_{\tau}\right\rfloor
$$

donde $\left(\delta(\tau) \mid F_{t}\right)$ es un valor conocido en $t$.

Descomponiendo el sumatorio de la expresión (10), en dos componentes en función del momento $t$ de valoración, tenemos,

$$
\sum_{\varphi=0}^{T^{\prime}-\tau} \Delta_{\varphi} Y_{\tau}=\sum_{\varphi=0}^{t-\tau} \Delta_{\varphi} Y_{\tau}+\sum_{\varphi=t+1-\tau}^{T^{\prime}-\tau} \Delta_{\varphi} Y_{\tau}
$$

y sustituyendo este resultado en la expresión (10), se obtiene, 


$$
\begin{aligned}
L I_{1}\left(T^{\prime}\right) & =\frac{1}{c t e} \sum_{\tau=1}^{t}\left(\delta(\tau) \mid F_{t}\right)\left[\sum_{\varphi=0}^{T^{\prime}-\tau} \Delta_{\varphi} Y_{\tau}\right]=\frac{1}{c t e} \sum_{\tau=1}^{t}\left(\delta(\tau) \mid F_{t}\right)\left[\sum_{\varphi=0}^{t-\tau} \Delta_{\varphi} Y_{\tau}+\sum_{\varphi=t+1-\tau}^{T^{\prime}-\tau} \Delta_{\varphi} Y_{\tau}\right]= \\
& =\frac{1}{c t e}\left[\sum_{\tau=1}^{t}\left(\delta(\tau) \mid F_{t}\right)\left(\sum_{\varphi=0}^{t-\tau} \Delta_{\varphi} Y_{\tau}\right)+\sum_{\tau=1}^{t}\left(\delta(\tau) \mid F_{t}\right)\left(\sum_{\varphi=t+1-\tau}^{T^{\prime}-\tau} \Delta_{\varphi} Y_{\tau}\right)\right]
\end{aligned}
$$

donde $\sum_{\tau=1}^{t}\left(\delta(\tau) \mid F_{t}\right)\left(\sum_{\varphi=0}^{t-\tau} \Delta_{\varphi} Y_{\tau}\right)=L I(t)$.

Por tanto:

$$
L I_{1}\left(T^{\prime}\right)=\frac{1}{c t e}\left[L I(t)+\sum_{\tau=1}^{t}\left(\delta(\tau) \mid F_{t}\right)\left(\sum_{\varphi=t+1-\tau}^{T^{\prime}-\tau} \Delta_{\varphi} Y_{\tau}\right)\right]
$$

La componente $L I_{2}\left(T^{\prime}\right)$, que no depende de la cuantía $L I(t)$, tiene la siguiente expresión:

$$
L I_{2}\left(T^{\prime}\right)=\frac{1}{c t e}\left[\sum_{\tau=t+1}^{T} \delta(\tau)\left(\sum_{\varphi=0}^{T^{\prime}-\tau} \Delta_{\varphi} Y_{\tau}\right)\right]
$$

Entonces, la variable aleatoria condicionada, $\operatorname{LI}\left(T^{\prime}\right) \mid F_{t}$, resulta de sustituir las expresiones (12) y (13) en la igualdad (9):

$$
\begin{aligned}
& L I\left(T^{\prime}\right) \mid F_{t}=\frac{1}{c t e}\left[L I(t)+\sum_{\tau=1}^{t}\left(\delta(\tau) \mid F_{t}\right)\left(\sum_{\varphi=t+1-\tau}^{T^{\prime}-\tau} \Delta_{\varphi} Y_{\tau}\right)+\sum_{\tau=t+1}^{T} \delta(\tau)\left(\sum_{\varphi=0}^{T^{\prime}-\tau} \Delta_{\varphi} Y_{\tau}\right)\right] \\
& \text { - Si } t \in\left\{T+1, T+2, \ldots, T^{\prime}\right\}, \\
& L I\left(T^{\prime}\right) \mid F_{t}=L I_{1}\left(T^{\prime}\right)
\end{aligned}
$$


por tanto:

$$
L I\left(T^{\prime}\right) \mid F_{t}=\frac{1}{c t e}\left[L I(t)+\sum_{\tau=1}^{T}\left(\delta(\tau) \mid F_{t}\right)\left(\sum_{\varphi=t+1-\tau}^{T^{\prime}-\tau} \Delta_{\varphi} Y_{\tau}\right)\right]
$$

\section{CONCRECIÓN DEL MODELO. SUSTITUCIÓN DE LOS INCREMENTOS $\Delta_{\varphi} Y_{\tau}$}

En el modelo discreto cierto utilizado para la determinación de la ratio de siniestralidad, suponemos que la cuantía de siniestros pendiente de declarar en $t, R_{\tau}(t)$, decrece proporcionalmente en el tiempo a razón de una tasa constante que denominamos tasa nominal de declaración de siniestros (que en el caso de periodos unitarios coincide con la tasa efectiva de declaración de siniestros), y que funciona como un tanto nominal de descuento.

Bajo esta hipótesis, la dinámica de la cuantía de siniestros pendiente de declarar en el momento $t, R_{\tau}(t)$, podemos representarla a través de la siguiente ecuación en diferencias finitas cierta:

$$
\Delta R_{\tau}(t)=-\alpha R_{\tau}(t) \Delta t
$$

Resolvemos esta ecuación en diferencias finitas cierta, de primer orden, con coeficientes constantes y homogénea, considerando periodos unitarios, $\Delta t=1$, de forma que,

$$
R_{\tau}(t+1)-R_{\tau}(t)=-\alpha R_{\tau}(t) \Rightarrow R_{\tau}(t+1)-(1-\alpha) R_{\tau}(t)=0
$$

donde comprobamos que $R_{\tau}(t)=z^{t}$ y $R_{\tau}(t+1)=z^{t+1}$, es una solución particular de la ecuación,

$$
\begin{aligned}
& z^{t+1}-(1-\alpha) z^{t}=0 \\
& z^{t}[z-(1-\alpha)]=0 \\
& z=(1-\alpha)
\end{aligned}
$$

Por tanto, la solución particular de la ecuación en diferencias finitas cierta resulta,

$$
R_{\tau}(t)=(1-\alpha)^{t}
$$


y la solución general es,

$$
R_{\tau}(t)=R_{\tau}(\tau)(1-\alpha)^{t}
$$

donde $R_{\tau}(\tau)$ es la cuantía de siniestros pendiente de declarar en el momento en que se produce la catástrofe y, por tanto, coincide con el volumen total de la misma, $R_{\tau}(\tau)=K_{\tau}$ (condición de contorno inicial).

Por tanto, la cuantía de siniestros pendiente de declarar al final del periodo $[t-1, t]$ resulta:

$$
R_{\tau}(t)=K_{\tau}(1-\alpha)^{t}
$$

Una vez determinada la forma de la variable $R_{\tau}(t)$, obtenemos el valor de sus incrementos,

$$
\begin{aligned}
\Delta R_{\tau}(t) & =R_{\tau}(t+1)-R_{\tau}(t)=K_{\tau}(1-\alpha)^{t+1}-K_{\tau}(1-\alpha)^{t}= \\
& =K_{\tau}(1-\alpha)^{t}[(1-\alpha)-1]=-\alpha K_{\tau}(1-\alpha)^{t}
\end{aligned}
$$

cuyo valor es negativo porque $R_{\tau}(t)$ es una función decreciente en el tiempo.

A partir de la definición de la cuantía total de una catástrofe, $K_{\tau}$ como suma de las variables $S_{\tau}(t)$ y $R_{\tau}(t)$ obtenemos la cuantía declarada de siniestros hasta $t, S_{\tau}(t)$, sustituyendo la variable $R_{\tau}(t)$ por su valor dado en la expresión (17) y operando:

$$
S_{\tau}(t)=K_{\tau}-R_{\tau}(t)=K_{\tau}-K_{\tau}(1-\alpha)^{t}=K_{\tau}\left[1-(1-\alpha)^{t}\right]
$$

El valor de los incrementos para la cuantía declarada de siniestros hasta $t$, resulta:

$$
\begin{aligned}
\Delta S_{\tau}(t) & =S_{\tau}(t+1)-S_{\tau}(t)=K_{\tau}\left[1-(1-\alpha)^{t+1}\right]-K_{\tau}\left[1-(1-\alpha)^{t}\right]= \\
& =K_{\tau}\left[(1-\alpha)^{t}-(1-\alpha)^{t+1}\right]=K_{\tau}(1-\alpha)^{t}[1-(1-\alpha)]=K_{\tau} \alpha(1-\alpha)^{t}
\end{aligned}
$$

Determinado el valor de los incrementos de las variables $R_{\tau}(t)$ y $S_{\tau}(t)$ obtenemos las expresiones finales de estas variables en función de dichos incrementos, sustituyendo los resultados (19) y (20) en las expresiones (3) y (2) respectivamente de la sección 2 anterior, de donde resultan: 


$$
\begin{aligned}
& R_{\tau}(t)=\delta(\tau)\left[K_{\tau}\left[1-\sum_{\varphi=0}^{t-\tau} \alpha(1-\alpha)^{\varphi}\right]\right] \\
& S_{\tau}(t)=\delta(\tau)\left[K_{\tau} \sum_{\varphi=0}^{t-\tau} \alpha(1-\alpha)^{\varphi}\right]
\end{aligned}
$$

Los resultados obtenidos en las expresiones (21) y (22) para la cuantía de siniestros pendiente de declarar y para la cuantía de siniestros declarada, $R_{\tau}(t)$ y $S_{\tau}(t)$, nos permiten reescribir todas las variables que intervienen en el proceso desarrollado para la determinación de la ratio de siniestralidad.

Entonces, la cuantía acumulada de declaraciones de pérdidas al final del periodo $T^{\prime}$ resulta:

$$
L I\left(T^{\prime}\right)=\frac{1}{c t e} \sum_{\tau=1}^{T^{\prime}} S_{\tau}\left(T^{\prime}\right)=\sum_{\tau=1}^{T} \delta(\tau)\left\{K_{\tau} \sum_{\varphi=0}^{T^{\prime}-\tau} \alpha(1-\alpha)^{\varphi}\right]
$$

A continuación, calculamos el valor de la cuantía total de declaraciones de pérdidas en $T^{\prime}$ condicionada a la información disponible sobre catástrofes en el momento $t, L I\left(T^{\prime}\right) \mid F_{t}$, para lo cual necesitamos, previamente, determinar el valor de su condicionante, esto es, la cuantía total declarada de siniestros hasta ese momento, $L I(t)$.

La expresión de $L I(t)$ presenta dos alternativas en función de dónde se sitúe el momento $t$ de valoración:

$$
\begin{aligned}
& \text { Si } t \in\{1,2, \ldots, T\}, \\
& L I(t)=\sum_{\tau=1}^{t} \delta(\tau)\left[K_{\tau} \sum_{\varphi=0}^{t-\tau} \alpha(1-\alpha)^{\varphi}\right] \\
& \text { Si } t \in\left\{T+1, T+2, \ldots, T^{\prime}\right\}, \\
& L I(t)=\sum_{\tau=1}^{t} \delta(\tau)\left[K_{\tau} \sum_{\varphi=0}^{t-\tau} \alpha(1-\alpha)^{\varphi}\right]
\end{aligned}
$$


En general, para cualquier $t \in\left\{1,2, \ldots, T, T+1, T+2, \ldots, T^{\prime}\right\}$ tenemos:

$$
L I(t)=\sum_{\tau=1}^{\min \{t, T\}} \delta(\tau)\left[K_{\tau} \sum_{\varphi=0}^{t-\tau} \alpha(1-\alpha)^{\varphi}\right]
$$

Siguiendo el proceso desarrollado en el epígrafe anterior, una vez determinada la cuantía $L I(t)$ la incorporamos en la variable $L I\left(T^{\prime}\right)$, descomponiendo la variable condicionada $L I\left(T^{\prime}\right) \mid F_{t}$ en la suma de dos variables independientes. De esta forma:

Si $t \in\{1,2, \ldots, T\}$,

$$
L I\left(T^{\prime}\right) \mid F_{t}=L I_{1}\left(T^{\prime}\right)+L I_{2}\left(T^{\prime}\right)
$$

donde:

$$
L I_{1}\left(T^{\prime}\right)=\frac{1}{c t e}\left[\sum_{\tau=1}^{t}\left(\delta(\tau) \mid F_{t}\right)\left(\left(K_{\tau} \mid F_{t}\right) \sum_{\varphi=0}^{T^{\prime}-\tau} \alpha(1-\alpha)^{\varphi}\right)\right]
$$

Descomponiendo el sumario de la expresión (28) en dos,

$$
\sum_{\varphi=0}^{T^{\prime}-\tau} \alpha(1-\alpha)^{\varphi}=\sum_{\varphi=0}^{t-\tau} \alpha(1-\alpha)^{\varphi}+\sum_{\varphi=t+1-\tau}^{T^{\prime}-\tau} \alpha(1-\alpha)^{\varphi}
$$

y substituyendo el resultado en la expresión (28), obtenemos:

$$
\left.L I_{1}\left(T^{\prime}\right)=\frac{1}{c t e}\left[L I(t)+\sum_{\tau=1}^{t}\left(\delta(\tau) \mid F_{t}\right)\left(K_{\tau} \mid F_{t}\right) \sum_{\varphi=t+1-\tau}^{T^{\prime}-\tau} \alpha(1-\alpha)^{\varphi}\right)\right]
$$

La componente $\mathrm{LI}_{2}\left(T^{\prime}\right)$ la calculamos como sigue:

$$
L I_{2}\left(T^{\prime}\right)=\frac{1}{c t e}\left[\sum_{\tau=t+1}^{T} \delta(\tau)\left(K_{\tau} \sum_{\varphi=0}^{T^{\prime}-\tau} \alpha(1-\alpha)^{\varphi}\right)\right]
$$


A partir de las ecuaciones (29) y (30), sustituyéndolas en la expresión (27), determinamos la variable aleatoria condicionada $L I\left(T^{\prime}\right) \mid F_{t}$ para cualquier momento de valoración perteneciente al periodo de riesgo:

$$
\begin{aligned}
& L I\left(T^{\prime}\right) \mid F_{t}=L I_{1}\left(T^{\prime}\right)+L I_{2}\left(T^{\prime}\right)= \\
& =\frac{1}{c t e}\left[L I(t)+\sum_{\tau=1}^{t}\left(\delta(\tau) \mid F_{t}\right)\left(\left(K_{\tau} \mid F_{t}\right) \sum_{\varphi=t+1-\tau}^{T^{\prime}-\tau} \alpha(1-\alpha)^{\varphi}\right)+\sum_{\tau=t+1}^{T} \delta(\tau)\left(K_{\tau} \sum_{\varphi=0}^{T^{\prime}-\tau} \alpha(1-\alpha)^{\varphi}\right)\right](31, \\
& -\quad \text { Si } t \in\left\{T+1, T+2, \ldots, T^{\prime}\right\} \\
& L I\left(T^{\prime}\right) \mid F_{t}=L I_{1}\left(T^{\prime}\right)
\end{aligned}
$$

donde:

$$
L I_{1}\left(T^{\prime}\right)=\frac{1}{c t e}\left[L I(t)+\sum_{\tau=1}^{T}\left(\delta(\tau) \mid F_{t}\right)\left(\left(K_{\tau} \mid F_{t}\right) \sum_{\varphi=t+1-\tau}^{T^{\prime}-\tau} \alpha(1-\alpha)^{\varphi}\right)\right]
$$

\section{CONCLUSIONES}

En este artículo se plantea un modelo en tiempo discreto para determinar la evolución de la ratio de siniestralidad de los activos derivados vinculados a seguros. Como hipótesis central para llevar a cabo la sistematización del modelo discreto en un contexto cierto, consideramos que la cuantía de siniestros pendiente de declarar decrece en el tiempo de forma proporcional a una tasa que nombramos, en este caso, tasa nominal de declaración de siniestros. Dicha tasa nominal, funciona como un tanto nominal de descuento y coincide con la tasa efectiva de declaración de siniestros en el caso de periodos unitarios.

Concretamos la dinámica cierta de la variable $R_{\tau}(t)$ en la siguiente ecuación en diferencias finitas,

$$
\Delta R_{\tau}(t)=-\alpha R_{\tau}(t) \Delta t
$$

a partir de la cual obtenemos el valor de sus incrementos,

$$
\Delta R_{\tau}(t)=-K_{\tau} \alpha(1-\alpha)^{t}
$$


donde $K_{\tau}$ se define como la suma de todas las catástrofes ocurridas en los diferentes instantes temporales que integran el periodo considerado.

Basándonos en los incrementos de la cuantía de siniestros pendiente de declarar, obtenemos las expresión final de dicha magnitud:

$$
R_{\tau}(t)=\delta(\tau)\left[K_{\tau}\left[1-\sum_{\varphi=0}^{t-\tau} \alpha(1-\alpha)^{\varphi}\right]\right]
$$

A partir de esta cuantía, el total de siniestros declarados se obtiene por diferencia entre la cuantía total de la catástrofe y la cuantía de siniestros pendiente de declarar:

$$
S_{\tau}(t)=\delta(\tau)\left[K_{\tau} \sum_{\varphi=0}^{t-\tau} \alpha(1-\alpha)^{\varphi}\right]
$$

La cuantía total de las pérdidas en $T^{\prime}$, numerador de la ratio de pérdidas que queremos calcular, se obtiene por agregación de la cuantía declarada de siniestros individualmente para cada una de las catástrofes sobrevenidas:

$$
L I\left(T^{\prime}\right)=\frac{1}{c t e} \sum_{\tau=1}^{T^{\prime}} S_{\tau}\left(T^{\prime}\right)=\sum_{\tau=1}^{T} \delta(\tau)\left[K_{\tau} \sum_{\varphi=0}^{T^{\prime}-\tau} \alpha(1-\alpha)^{\varphi}\right]
$$

\section{BIBLIOGRAFÍA}

Aase, K., "An Equilibrium Model of Catastrophe Insurance Futures and Spreads," Geneva Papers on Risk and Insurance Theory, 24, 1999, pp. 69-96.

AASE, K., "A Markov model for the pricing of catastrophe insurance futures and spreads," Journal of Risk and Insurance, vol. 68 (1), 2001, pp. 25-50.

Alegre, A, M. J. Pérez-Fructuoso y P. Devolder, "Modèles discrets d'options sur risques catastrophiques," Belgian Actuarial Bulletin, 3, 2003, pp. 28-32.

Baryshnikov, Y., A. Mayo y D. R. Taylor, "Pricing of Cat Bonds," Working Paper, Version October 3, 2001.

Biagini, F., Y. Bregman y T. Meyer-Brandis, "Pricing of catastrophe insurance options written on a loss index with reestimation," Insurance: Mathematics and Economics 43, 2008, pp. 214-222. 
Burnecki, K. y G. Kukla, "Pricing of zero-coupon and coupon cat bonds," Applicationes Mathematicae, 30, 2003, pp. 315-324.

Burnecki, K., Pricing catastrophe bonds in a compound non-homogeneous Poisson model with left truncated loss distributions. Presentation Wroclaw University of Technology, 2005.

Cummins, J. D. y H. Geman, "Pricing Catastrophe Insurance Futures and Call Spreads: An Arbitrage Approach," Journal of Fixed Income, 4, 1995, pp. 46-57.

Cox, S. H. y H. Pedersen, "Catastrophe Risk Bonds," North American Actuarial Journal, 4 (4), 2000, pp. 56-82.

Egami, M. y V. Young, "Indifference prices of structured catastrophe (CAT) bonds," Insurance: Mathematics and Economics, 42, 2008, pp. 771-778.

Embrechts, P., y S. Meister, Pricing insurance derivatives, the case of CAT futures. Proceedings of the 1995 Bowles Symposium on Securitization of Insurance Risk, Georgia State University, Atlanta, Georgia. Society of Actuaries, Monograph M-FI97-1: 15-26, 1997.

Geman, H. y M. Yor, "Stochastic time changes in catastrophe option pricing," Insurance: Mathematics and economics, 21, 1997, pp. 185-193.

LAI, V. S, M. PARcollet y B.F. Lamond, "The valuation of catastrophe bonds with exposure to currency exchange risk," International Review of Financial Analysis, 33, 2014, pp. 243-252.

LATORRE, L. Teoría del riesgo y sus aplicaciones a la empresa aseguradora. Ed. Mapfre S.A. Madrid. 1992

LEE, J.P. y M. T. Yu, "Pricing default-risky Cat bonds with moral hazard and basis risk," Journal of Risk and Insurance, 69 (1), 2002, pp. 25-44.

LEE, J.P. y M. T. Yu, "Valuation of catastrophe reinsurance with catastrophe bonds," Insurance: Mathematics and Economics, 41 (2), 2007, pp. 264-278.

Muermann, A., Actuarially Consistent Valuation of Catastrophe Derivatives, Working Paper Series The Wharton Financial Institutions Center, 03-18, 2003.

NowAK, P. y M. Romaniuk, "Princing and simulations of catastrophe Bonds," Insurance: Mathematics and Economics, 52, 2013, pp. 18-28.

PAnJer, H. Financial Economics, The Actuarial Foundation. Illinois. 1998.

PÉrez-Fructuoso, M.J., "Elaborating a catastrophic loss index for insurance-linked securities (ILS) a continuous model," Asian-Pacific Journal of Risk and Insurance, 3 (2), 2009, pp. 34-45.

Pérez-Fructuoso, M. J., "A continuous model to calculate the loss trigger index of a CAT Bond," Variance, 2 (2), 2008, pp. 253-265. 
Pérez-Fructuoso, M. J., "La titulización del riesgo catastrófico: descripción y análisis de los cat bonds (Bonos de Catástrofes)," Revista Española de Seguros, 121, 2005, pp. 75-92.

Unger, A.J.A., "Pricing index-based catastrophe bonds: Part 1. Formulation and discretization issues using a numerical PDE approach," Computers \& Geosciences, 36, 2010, pp. 139-149.

Zong-Gang, M. y Chao-Qun, M., "Princing catastrophe risk Bonds: a mixed approximation method," Insurance: Mathematics and Economics, 52, 2013, pp. 243254. 